\title{
Small-Angle Bhabha Scattering*
}

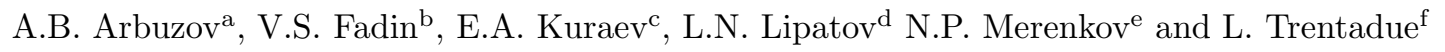 \\ a Joint Institute for Nuclear Research Dubna, Moscow region, 141980, Russia \\ b Budker Institute for Nuclear Physics Novosibirsk State University, 630090, Novosibirsk, Russia \\ ${ }^{\mathrm{c} J o i n t ~ I n s t i t u t e ~ f o r ~ N u c l e a r ~ R e s e a r c h ~ D u b n a, ~ M o s c o w ~ r e g i o n, ~ 141980, ~ R u s s i a ~}$ \\ ${ }^{d}$ St.-Petersburg Institute of Nuclear Physics, Gatchina, Leningrad region, 188350, Russia \\ ePhysics-Techn. Institute, Kharkov, Ukraine \\ ${ }^{\mathrm{f}}$ Dipartimento di Fisica, Universitá di Parma and INFN, Gruppo Collegato di Parma, Parma, Italy.
}

We present the calculation of the elastic and inelastic high-energy small-angle electron-positron scattering with a per mille accuracy.

\section{Introduction}

A considerable attention has been recently devoted to the Bhabha process [1-3]. The reached accuracy is however still inadequate [5] with respect to the experimental one 四. According to these evaluations the theoretical estimates are still incomplete; moreover, they are somewhat larger $(\sim$ a factor 2$)$ than the projected theoretical and experimental precision [5] and are comparable to the currently published experimental precision.

The process that will be considered in this work is that of Bhabha scattering when electrons and positrons are emitted at small angles with respect to the initial electron and positron directions. We have examined the radiative processes inclusively accompanying the main $\mathrm{e}^{+} \mathrm{e}^{-} \rightarrow \mathrm{e}^{+} \mathrm{e}^{-}$reaction at high energies, when both the scattered electron and positron are tagged within the counter aperture.

We assume that the center-of-mass energies are within the range of the LEP collider $2 \epsilon=$ $\sqrt{s}=90-200 \mathrm{GeV}$ and the scattering angles are within the range $\theta \simeq 10-150 \mathrm{mrad}$. We assume that the charged-particle detectors have

\footnotetext{
${ }^{*}$ Invited talk presented by L. Trentadue at the Zeuthen workshop on Elementary Particle Theory "QCD and QED in Higher Orders", Rheinsberg, April 21-26, 1996.
}

the following polar angle cuts:

$$
\begin{aligned}
& \theta_{1}<\theta_{-}={\widehat{\boldsymbol{p}_{1} \boldsymbol{q}}}_{1} \equiv \theta<\theta_{3}, \\
& \theta_{2}<\theta_{+}=\widehat{\boldsymbol{p}}_{2} \boldsymbol{q}_{2}<\theta_{4}, \\
& 0.01 \lesssim \theta_{i} \lesssim 0.1 \mathrm{rad},
\end{aligned}
$$

where $\boldsymbol{p}_{1}, \boldsymbol{q}_{1}\left(\boldsymbol{p}_{2}, \boldsymbol{q}_{2}\right)$ are the momenta of the initial and of the scattered electron (positron) in the center-of-mass frame.

In this talk we present the results of our calculations of the electron-positron scattering cross-section with an accuracy of $\mathcal{O}(0.1 \%)$. The squared matrix elements of the various exclusive processes inclusively contributing to the $e^{+} e^{-} \rightarrow$ $e^{+} e^{-}$reaction are integrated in order to define an experimentally measurable cross-section according to suitable restrictions on the angles and energies of the detected particles. The different contributions to the electron and positron distributions, needed for the required accuracy, are presented using analytical expressions.

In order to define the angular range of interest and the implications on the required accuracy, let us first briefly discuss, in a general way, the angledependent corrections to the cross-section.

We consider $e^{+} e^{-}$scattering at angles as defined in Eq. (11). Within this region, if one expresses the cross-section by means of a series ex- 
pansion in terms of angles, the main contribution to the cross-section $\mathrm{d} \sigma / \mathrm{d} \theta^{2}$ comes from the diagrams for the scattering amplitudes containing one exchanged photon in the $t$-channel. These diagrams, as well known, show a singularity of the type $\theta^{-4}$ for $\theta \rightarrow 0$, e.g.

$\frac{\mathrm{d} \sigma}{\mathrm{d} \theta^{2}} \sim \theta^{-4}$.

Let us now estimate the correction of order $\theta^{2}$ to this contribution. If

$\frac{\mathrm{d} \sigma}{\mathrm{d} \theta^{2}} \sim \theta^{-4}\left(1+c_{1} \theta^{2}\right)$,

then, after integration over $\theta^{2}$ in the angular range of Eq. (11), we obtain:

$$
\int_{\theta_{\min }^{2}}^{\theta_{\max }^{2}} \frac{\mathrm{d} \sigma}{\mathrm{d} \theta^{2}} \mathrm{~d} \theta^{2} \sim \theta_{\min }^{-2}\left(1+c_{1} \theta_{\min }^{2} \ln \frac{\theta_{\max }^{2}}{\theta_{\min }^{2}}\right) .
$$

We see that, for $\theta_{\min }=50 \mathrm{mrad}$ and $\theta_{\max }=$ $150 \mathrm{mrad}$ (we have taken the case where the $\theta^{2}$ corrections are maximal), the relative contribution of the $\theta^{2}$ terms is about $5 \times 10^{-3} c_{1}$. Therefore, the terms of relative order $\theta^{2}$ must be kept only in the Born cross-section where the coefficient $c_{1}$ is not small.

A detailed derivation of these results has been reported elsewhere (see [7] and references therein).

\section{Born cross-section and one-loop virtual and soft corrections}

The Born cross-section for Bhabha scattering within the Standard Model is well known 33. In the small-angle limit is

$\frac{\mathrm{d} \sigma^{B}}{\theta \mathrm{d} \theta}=\frac{8 \pi \alpha^{2}}{\varepsilon^{2} \theta^{4}}\left(1-\frac{\theta^{2}}{2}+\frac{9}{40} \theta^{4}+\delta_{\text {weak }}\right)$,

where $\varepsilon=\sqrt{s} / 2$ is the electron or positron initial energy and the weak correction term $\delta_{\text {weak }}$, connected with diagrams with $Z^{0}$-boson exchange.

In the pure QED case one-loop radiative corrections to Bhabha cross-section were calculated a long time ago 8. Taking into account a contribution from soft-photon emission with energy less than a given finite threshold $\Delta \varepsilon$, we have here for the cross-section $d \sigma_{Q E D}^{(1)}$, in the one-loop approximation for the limiting case of small scattering angles:

$$
\begin{aligned}
& \frac{\mathrm{d} \sigma_{Q E D}^{(1)}}{\mathrm{d} c}=\frac{\mathrm{d} \sigma_{Q E D}^{B}}{\mathrm{~d} c}(1-\Pi(t))^{-2}(1+\delta), \\
& \delta=2 \frac{\alpha}{\pi}\left[2(1-L) \ln \frac{1}{\Delta}+\frac{3}{2} L-2\right] \\
& +\frac{\alpha}{\pi} \theta^{2} \Delta_{\theta}+\frac{\alpha}{\pi} \theta^{2} \ln \Delta, \\
& \Delta_{\theta}=\frac{3}{16} l^{2}+\frac{7}{12} l-\frac{19}{18}+\frac{1}{4}\left(\delta_{t}-\delta_{s}\right), \\
& \Delta=\frac{\Delta \varepsilon}{\varepsilon}, \quad l=\ln \frac{Q^{2}}{s} \simeq \ln \frac{\theta^{2}}{4} .
\end{aligned}
$$

The photon vacuum polarization function $\Pi(t)$ $(\Pi(s))$ is defined as follows:

$\Pi(t)=\frac{\alpha}{\pi}\left(\delta_{t}+\frac{1}{3} L-\frac{5}{9}\right)+\frac{1}{4}\left(\frac{\alpha}{\pi}\right)^{2} L$,

where

$L=\ln \frac{Q^{2}}{m^{2}}, \quad Q^{2}=-t=2 \varepsilon^{2}(1-c)$,

and we took into account the leading part of the two-loop contribution in the polarization operator. In the Standard Model, $\delta_{t}$ contains contributions of muons, tau-leptons, $W$-bosons and hadrons. For numerical calculations we use for $\Pi(t)$ the results of Ref. [9].

Taking into account that the large contribution proportional to $\ln \Delta$ disappears when we add the cross-section for the hard emission, one can verify that terms of relative order $\theta^{2}$ can be neglected. Therefore we will omit in higher orders the annihilation diagrams and multiple-photon exchange diagrams in the scattering channel. The second simplification is justified by the generalized eikonal representation for small-angle scattering amplitudes. In particular, for the case of elastic processes we have [10]:

$$
\begin{aligned}
& A(s, t)=A_{0}(s, t) F_{1}^{2}(t)(1-\Pi(t))^{-1} \mathrm{e}^{i \varphi(t)} \\
& \times\left[1+\mathcal{O}\left(\frac{\alpha}{\pi} \frac{Q^{2}}{s}\right)\right], \quad s \gg Q^{2} \gg m^{2},
\end{aligned}
$$


where $A_{0}(s, t)$ is the Born amplitude, $F_{1}(t)$ is the Dirac form factor and $\varphi(t)=-\alpha \ln \left(Q^{2} / \lambda^{2}\right)$ is the Coulomb phase, $\lambda$ is the photon mass auxiliary parameter. We may consider the eikonal representation as correct within the required accuracy $2^{2}$.

Let us now introduce the dimensionless quantity $\Sigma=Q_{1}^{2} \sigma_{\exp } /\left(4 \pi \alpha^{2}\right)$, with $Q_{1}^{2}=\varepsilon^{2} \theta_{1}^{2}$, where $\sigma_{\exp }$ is the Bhabha-process cross-section integrated over the typical experimental energy and angular ranges 3 :

$$
\begin{aligned}
\Sigma & =\frac{Q_{1}^{2}}{4 \pi \alpha^{2}} \int \mathrm{d} x_{1} \int \mathrm{d} x_{2} \Theta\left(x_{1} x_{2}-x_{c}\right) \\
& \times \int \mathrm{d}^{2} \boldsymbol{q}_{1}^{\perp} \Theta_{1}^{c} \int \mathrm{d}^{2} \boldsymbol{q}_{2}^{\perp} \Theta_{2}^{c} \\
& \times \frac{\mathrm{d} \sigma^{e^{+} e^{-} \rightarrow e^{+}\left(\boldsymbol{q}_{2}^{\perp}, x_{2}\right) e^{-}\left(\boldsymbol{q}_{1}^{\perp}, x_{1}\right)+X}}{\mathrm{~d} x_{1} \mathrm{~d}^{2} \boldsymbol{q}_{1}^{\perp} \mathrm{d} x_{2} \mathrm{~d}^{2} \boldsymbol{q}_{2}^{\perp}}
\end{aligned}
$$

where $x_{1,2}, \boldsymbol{q}_{1,2}^{\perp}$ are the energy fractions and the transverse components of the momenta of the electron and positron in the final state, $s x_{c}$ is the experimental cut-off on their invariant mass squared and the functions $\Theta_{i}^{c}$ do take into account the angular cuts (11):

$$
\begin{aligned}
& \Theta_{1}^{c}=\Theta\left(\theta_{3}-\frac{\left|\boldsymbol{q}_{1}^{\perp}\right|}{x_{1} \varepsilon}\right) \Theta\left(\frac{\left|\boldsymbol{q}_{1}^{\perp}\right|}{x_{1} \varepsilon}-\theta_{1}\right), \\
& \Theta_{2}^{c}=\Theta\left(\theta_{4}-\frac{\left|\boldsymbol{q}_{2}^{\perp}\right|}{x_{2} \varepsilon}\right) \Theta\left(\frac{\left|\boldsymbol{q}_{2}^{\perp}\right|}{x_{2} \varepsilon}-\theta_{2}\right) .
\end{aligned}
$$

In the case of a symmetrical angular acceptance (we restrict ourselves further to this case only) we have:

$\theta_{2}=\theta_{1}, \quad \theta_{4}=\theta_{3}, \quad \rho=\frac{\theta_{3}}{\theta_{1}}>1$.

We will present $\Sigma$ as the sum of various contributions:

$$
\begin{aligned}
\Sigma & =\Sigma_{0}+\Sigma^{\gamma}+\Sigma^{2 \gamma}+\Sigma^{e^{+} e^{-}}+\Sigma^{3 \gamma}+\Sigma^{e^{+} e^{-} \gamma} \\
& =\Sigma_{00}\left(1+\delta_{0}+\delta^{\gamma}+\delta^{2 \gamma}+\delta^{e^{+} e^{-}}\right. \\
& \left.+\delta^{3 \gamma}+\delta^{e^{+} e^{-} \gamma}\right), \quad \Sigma_{00}=1-\rho^{-2}
\end{aligned}
$$

$\overline{2}$ Result obtained in paper [1], we believe, is incorrect. It contradicts to the well established result of D. Yennie et al. [12] about cancelation of infrared singularities.

${ }^{3}$ Really this quantity corresponds to some ideal detectors. It is intended for comparisons with the results of Monte Carlo event generators. where $\Sigma_{0}$ stands for a modified Born contribution, $\Sigma^{\gamma}$ for a contribution of one-photon emission (real and virtual) and so on. The values of the $\delta^{i}$ as function of $x_{c}$ are given in Table 1 (see below). We shall slightly modify the perturbation theory, using the full propagator for the $t$-channel photon, which takes into account the growth of the electric charge at small distances. By integrating with this convention, we obtain:

$\Sigma_{0}=\theta_{1}^{2} \int_{\theta_{1}^{2}}^{\theta_{2}^{2}} \frac{\mathrm{d} \theta^{2}}{\theta^{4}}(1-\Pi(t))^{-2}+\Sigma_{W}+\Sigma_{\theta}$,

where $\Sigma_{W}$ is the correction due to the weak interaction:

$\Sigma_{W}=\theta_{1}^{2} \int_{\theta_{1}^{2}}^{\theta_{2}^{2}} \frac{\mathrm{d} \theta^{2}}{\theta^{4}} \delta_{\text {weak }}$,

and the term $\Sigma_{\theta}$ comes from the expansion of the Born cross-section in powers of $\theta^{2}$,

$\Sigma_{\theta}=\theta_{1}^{2} \int_{1}^{\rho^{2}} \frac{\mathrm{d} z}{z\left(1-\Pi\left(-z Q_{1}^{2}\right)\right)^{2}}\left(-\frac{1}{2}+z \theta_{1}^{2} \frac{9}{40}\right)$.

The remaining contributions to $\Sigma$ in 12 are considered below.

\subsection{Single hard-photon emission}

In order to calculate the contribution to $\Sigma$ due to the hard-photon emission we start from the corresponding differential cross-section written in terms of energy fractions $x_{1,2}$ and transverse components $\boldsymbol{q}_{1,2}^{\perp}$ of the final particle momenta [13]:

$$
\begin{aligned}
& \frac{\mathrm{d} \sigma_{B}^{e^{+}} e^{-} \rightarrow e^{+} e^{-} \gamma}{\mathrm{d} x_{1} \mathrm{~d}^{2} \boldsymbol{q}_{1}^{\perp} \mathrm{d} x_{2} \mathrm{~d}^{2} \boldsymbol{q}_{2}^{\perp}}=\frac{2 \alpha^{3}}{\pi^{2}}\left(1+\mathcal{O}\left(\theta^{2}\right)\right) \\
& \times\left\{\frac{R\left(x_{1} ; \boldsymbol{q}_{1}^{\perp}, \boldsymbol{q}_{2}^{\perp}\right) \delta\left(1-x_{2}\right)}{\left(\boldsymbol{q}_{2}^{\perp}\right)^{4}\left(1-\Pi\left(-\left(\boldsymbol{q}_{2}^{\perp}\right)^{2}\right)\right)^{2}}\right. \\
& \left.+\frac{R\left(x_{2} ; \boldsymbol{q}_{2}^{\perp}, \boldsymbol{q}_{1}^{\perp}\right) \delta\left(1-x_{1}\right)}{\left(\boldsymbol{q}_{1}^{\perp}\right)^{4}\left(1-\Pi\left(-\left(\boldsymbol{q}_{1}^{\perp}\right)^{2}\right)\right)^{2}}\right\},
\end{aligned}
$$

where

$$
R\left(x ; \boldsymbol{q}_{1}^{\perp}, \boldsymbol{q}_{2}^{\perp}\right)=\frac{1+x^{2}}{1-x}\left[\frac{\left(\boldsymbol{q}_{2}^{\perp}\right)^{2}(1-x)^{2}}{d_{1} d_{2}}\right.
$$




$$
\begin{gathered}
\left.-\frac{2 m^{2}(1-x)^{2} x}{1+x^{2}} \frac{\left(d_{1}-d_{2}\right)^{2}}{d_{1}^{2} d_{2}^{2}}\right], \\
d_{1}=m^{2}(1-x)^{2}+\left(\boldsymbol{q}_{1}^{\perp}-\boldsymbol{q}_{2}^{\perp}\right)^{2}, \\
d_{2}=m^{2}(1-x)^{2}+\left(\boldsymbol{q}_{1}^{\perp}-x \boldsymbol{q}_{2}^{\perp}\right)^{2},
\end{gathered}
$$

and we use the full photon propagator for the $t$ channel photon. Performing a simple azimuthal angle integration of Eq. (16) we obtain for the hard-photon emission the contribution $\Sigma^{H}$ :

$$
\begin{aligned}
& \Sigma^{H}=\frac{\alpha}{\pi} \int_{x_{c}}^{1-\Delta} \mathrm{d} x \frac{1+x^{2}}{1-x} \int_{1}^{\rho^{2}} \frac{\mathrm{d} z}{z^{2}\left(1-\Pi\left(-z Q_{1}^{2}\right)\right)^{2}} \\
& \times\left\{\left[1+\Theta\left(x^{2} \rho^{2}-z\right)\right](L-1)+k(x, z)\right\}, \\
& k(x, z)=\frac{(1-x)^{2}}{1+x^{2}}\left[1+\Theta\left(x^{2} \rho^{2}-z\right)\right]+L_{1} \\
& +\Theta\left(x^{2} \rho^{2}-z\right) L_{2}+\Theta\left(z-x^{2} \rho^{2}\right) L_{3},
\end{aligned}
$$

where $L=\ln \left(z Q_{1}^{2} / m^{2}\right)$ and

$$
\begin{aligned}
& L_{1}=\ln \left|\frac{x^{2}(z-1)\left(\rho^{2}-z\right)}{(x-z)\left(x \rho^{2}-z\right)}\right|, \\
& L_{2}=\ln \left|\frac{\left(z-x^{2}\right)\left(x^{2} \rho^{2}-z\right)}{x^{2}(x-z)\left(x \rho^{2}-z\right)}\right|, \\
& L_{3}=\ln \left|\frac{\left(z-x^{2}\right)\left(x \rho^{2}-z\right)}{(x-z)\left(x^{2} \rho^{2}-z\right)}\right| .
\end{aligned}
$$

It is seen from Eq. (18) that $\Sigma^{H}$ contains the auxiliary parameter $\Delta$. This parameter disappears, as it should, in the sum $\Sigma^{\gamma}=\Sigma^{H}+\Sigma^{V+S}$, where $\Sigma^{V+S}$ is the contribution of virtual and soft real photons:

$$
\begin{aligned}
\Sigma^{\gamma} & =\frac{\alpha}{\pi} \int_{1}^{\rho^{2}} \frac{\mathrm{d} z}{z^{2}} \int_{x_{c}}^{1} \mathrm{~d} x\left(1-\Pi\left(-z Q_{1}^{2}\right)\right)^{-2} \\
& \times\left\{(L-1) P(x)\left[1+\Theta\left(x^{2} \rho^{2}-z\right)\right]\right. \\
& \left.+\frac{1+x^{2}}{1-x} k(x, z)-\delta(1-x)\right\},
\end{aligned}
$$

where is the non-singlet splitting kernel.

\section{Radiative corrections to $\mathcal{O}\left(\alpha^{2}\right)$}

A systematic treatment of all $\mathcal{O}\left(\alpha^{2}\right)$ contributions is absent up to now. This is mainly due to the extreme complexity of the analysis (more then 100 Feynman diagrams are to be taken into account considering elastic and inelastic processes). Nevertheless in the case of small scattering angles we may restrict ourselves by considering only diagrams of the scattering type.

\subsection{Virtual and soft corrections to the hard-photon emission}

By evaluating the corrections arising from the emission of virtual and real soft photons which accompany a single hard-photon we will consider two cases. The first case corresponds to the emission of the photons by the same fermion. The second one occurs when the hard-photon is emitted by another fermion:

$$
\begin{aligned}
\left.\mathrm{d} \sigma\right|_{H(S+V)} & =\mathrm{d} \sigma^{H(S+V)}+\mathrm{d} \sigma_{H(S+V)} \\
& +\mathrm{d} \sigma_{(S+V)}^{H}+\mathrm{d} \sigma_{H}^{(S+V)} .
\end{aligned}
$$

In the case when both fermions emit, one finds that:

$$
\begin{aligned}
\Sigma_{(S+V)}^{H}+\Sigma_{H}^{(S+V)} & =2 \Sigma^{H}\left(\frac{\alpha}{\pi}\right)[(L-1) \ln \Delta \\
& \left.+\frac{3}{4} L-1\right]
\end{aligned}
$$

where $\Sigma^{H}$ is given in Eq. (18). A more complex expression arises when the radiative corrections are applied to the same fermion line. Here the cross-section may be expressed in terms of the Compton tensor with an off-shell photon [14], which describes the process

$\gamma^{*}(q)+e^{-}\left(p_{1}\right) \rightarrow e^{-}\left(q_{1}\right)+\gamma(k)+\left(\gamma_{\mathrm{soft}}\right)$.

The result has the form:

$$
\begin{aligned}
& \Sigma^{H(S+V)}=\Sigma_{H(S+V)}=\frac{1}{2}\left(\frac{\alpha}{\pi}\right)^{2} \int_{1}^{\rho^{2}} \frac{\mathrm{d} z}{z^{2}} \\
& \times \int_{x_{c}}^{1-\Delta} \frac{\mathrm{d} x\left(1+x^{2}\right)}{1-x} L\left\{\left(2 \ln \Delta-\ln x+\frac{3}{2}\right)\right. \\
& \times[(L-1)(1+\Theta)+k(x, z)]+\frac{1}{2} \ln ^{2} x
\end{aligned}
$$




$$
\begin{aligned}
& +(1+\Theta)[-2+\ln x-2 \ln \Delta]+(1-\Theta) \\
& \times\left[\frac{1}{2} L \ln x+2 \ln \Delta \ln x-\ln x \ln (1-x)-\ln ^{2} x\right. \\
& \left.-\mathrm{Li}_{2}(1-x)-\frac{x(1-x)+4 x \ln x}{2\left(1+x^{2}\right)}\right] \\
& \left.-\frac{(1-x)^{2}}{2\left(1+x^{2}\right)}\right\}, \quad \operatorname{Li}_{2}(x) \equiv-\int_{0}^{x} \frac{\mathrm{d} t}{t} \ln (1-t)
\end{aligned}
$$

where $k(x, z)$ is given in Eq. (18) and $\Theta \equiv$ $\Theta\left(x^{2} \rho^{2}-z\right)$.

\subsection{Double hard-photon bremsstrahlung}

We now consider the contribution given by the process of emission of two hard photons. We will distinguish two cases: a) the double simultaneous bremsstrahlung in opposite directions along electron and positron momenta, and b) the double bremsstrahlung in the same direction along electron or positron momentum. The differential cross-section in the first case can be obtained by using the factorization property of cross-sections within the impact parameter representation [15]. It takes the following form [13]:

$$
\begin{aligned}
& \frac{\mathrm{d} \sigma^{e^{+} e^{-} \rightarrow\left(e^{+} \gamma\right)\left(e^{-} \gamma\right)}}{\mathrm{d} x_{1} \mathrm{~d}^{2} \boldsymbol{q}_{1}^{\perp} \mathrm{d} x_{2} \mathrm{~d}^{2} \boldsymbol{q}_{2}^{\perp}}=\frac{\alpha^{4}}{\pi^{3}} \int \frac{\mathrm{d}^{2} \boldsymbol{k}^{\perp}}{\pi\left(\boldsymbol{k}^{\perp}\right)^{4}} \\
& \times \frac{R\left(x_{1} ; \boldsymbol{q}_{1}^{\perp}, \boldsymbol{k}^{\perp}\right) R\left(x_{2} ; \boldsymbol{q}_{2}^{\perp},-\boldsymbol{k}^{\perp}\right)}{\left(1-\Pi\left(-\left(\boldsymbol{k}^{\perp}\right)^{2}\right)\right)^{2}},
\end{aligned}
$$

where $R\left(x ; \boldsymbol{q}^{\perp}, \boldsymbol{k}^{\perp}\right)$ is given by Eq. (17). The calculation of the corresponding contribution $\Sigma_{H}^{H}$ to $\Sigma$ is analogous to the case of the single hardphoton emission and the result has the form:

$$
\begin{aligned}
& \Sigma_{H}^{H}=\frac{1}{4}\left(\frac{\alpha}{\pi}\right)^{2} \int_{0}^{\infty} \frac{\mathrm{d} z}{z^{2}\left(1-\Pi\left(-z Q_{1}^{2}\right)\right)^{2}} \int_{x_{c}}^{1-\Delta} \mathrm{d} x_{1} \\
& \times \int_{x_{c} / x_{1}}^{1-\Delta} \mathrm{d} x_{2} \frac{1+x_{1}^{2}}{1-x_{1}} \frac{1+x_{2}^{2}}{1-x_{2}} \Phi\left(x_{1}, z\right) \Phi\left(x_{2}, z\right),
\end{aligned}
$$

where (see Eq. (19)):

$$
\begin{aligned}
& \Phi(x, z)=(L-1)\left[\Theta(z-1) \Theta\left(\rho^{2}-z\right)\right. \\
& \left.+\Theta\left(z-x^{2}\right) \Theta\left(\rho^{2} x^{2}-z\right)\right] \\
& +L_{3}\left[-\Theta\left(x^{2}-z\right)+\Theta\left(z-x^{2} \rho^{2}\right)\right]
\end{aligned}
$$

$$
\begin{aligned}
& +\left(L_{2}+\frac{(1-x)^{2}}{1+x^{2}}\right) \Theta\left(z-x^{2}\right) \Theta\left(x^{2} \rho^{2}-z\right) \\
& +\left(L_{1}+\frac{(1-x)^{2}}{1+x^{2}}\right) \Theta(z-1) \Theta\left(\rho^{2}-z\right) \\
& +\left(\Theta(1-z)-\Theta\left(z-\rho^{2}\right)\right) \ln \left|\frac{(z-x)\left(\rho^{2}-z\right)}{\left(x \rho^{2}-z\right)(z-1)}\right| .
\end{aligned}
$$

Let us now turn to the double hard-photon emission in the same direction and the hard $\mathrm{e}^{+}$ $\mathrm{e}^{-}$pair production. Here we use the method developed by one of us [16,17,. We will distinguish the collinear and semi-collinear kinematics of final particles. In the first case all produced particles move in the cones within the polar angles $\theta_{i}<\theta_{0} \ll 1$ centered along the charged-particle momenta (final or initial). In the semi-collinear region only one produced particle moves inside those cones, while the other moves outside them. For the totally inclusive cross-section, such a distinction no longer has physical meaning and the dependence on the auxiliary parameter $\theta_{0}$ disappears. We underline that in this way all double and single-logarithmical contributions may be extracted rigorously. The contribution of the region when both the photons move outside the small cones does not contain any large logarithm $L$. The systematic omission of those contributions in the double bremsstrahlung and pair production processes is the source of uncertainties of order $(\alpha / \pi)^{2} \leq 0.6 \cdot 10^{-5}$.

The contribution of both collinear and semicollinear regions (we consider for definiteness the emission of both hard photons along the electron, since the contribution of the emission along the positron is the same) has the form:

$$
\begin{aligned}
& \Sigma^{H H}=\Sigma_{H H}=\frac{1}{4}\left(\frac{\alpha}{\pi}\right)^{2} \int_{1}^{\rho^{2}} \frac{\mathrm{d} z}{z^{2}\left(1-\Pi\left(-z Q_{1}^{2}\right)\right)^{2}} \\
& \times \int_{x_{c}}^{1-2 \Delta} \mathrm{d} x \int_{\Delta}^{1-x-\Delta} \mathrm{d} x_{1} \frac{I^{H H} L}{x_{1}\left(1-x-x_{1}\right)\left(1-x_{1}\right)^{2}}, \\
& I^{H H}=A \Theta\left(x^{2} \rho^{2}-z\right)+B \\
& +C \Theta\left(\left(1-x_{1}\right)^{2} \rho^{2}-z\right),
\end{aligned}
$$

where $A, B$ and $C$ are known functions [7].

The total expression $\Sigma^{2 \gamma}$, which describes the contribution to (12) from the two-photon (real 
and virtual) emission processes reads as follows:

$$
\begin{aligned}
\Sigma^{2 \gamma} & =\Sigma_{S+V}^{\gamma \gamma}+2 \Sigma^{H(V+S)}+2 \Sigma_{S+V}^{H} \\
& +\Sigma_{H}^{H}+2 \Sigma^{H H} \\
& =\Sigma^{\gamma \gamma}+\Sigma_{\gamma}^{\gamma}+\left(\frac{\alpha}{\pi}\right)^{2} \mathcal{L}\left(\phi^{\gamma \gamma}+\phi_{\gamma}^{\gamma}\right) \\
\mathcal{L} & =\ln \frac{\varepsilon^{2} \theta_{1}^{2}}{m^{2}} .
\end{aligned}
$$

The leading contributions $\Sigma^{\gamma \gamma}, \Sigma_{\gamma}^{\gamma}$ have the following forms:

$$
\begin{aligned}
& \Sigma^{\gamma \gamma}=\frac{1}{2}\left(\frac{\alpha}{\pi}\right)^{2} \int_{1}^{\rho^{2}} \frac{\mathrm{d} z}{z^{2}} L^{2}\left(1-\Pi\left(-Q_{1}^{2} z\right)\right)^{-2} \\
& \times \int_{x_{c}}^{1} \mathrm{~d} x\left\{\frac{1}{2} P^{(2)}(x)\left[\Theta\left(x^{2} \rho^{2}-z\right)+1\right]\right. \\
& \left.+\int_{x}^{1} \frac{\mathrm{d} t}{t} P(t) P\left(\frac{x}{t}\right) \Theta\left(t^{2} \rho^{2}-z\right)\right\}, \\
& \Sigma_{\gamma}^{\gamma}=\frac{1}{4}\left(\frac{\alpha}{\pi}\right)^{2} \int_{0}^{\infty} \frac{\mathrm{d} z}{z^{2}} L^{2}\left(1-\Pi\left(-Q_{1}^{2} z\right)\right)^{-2} \\
& \times \int_{x_{c}}^{1} \mathrm{~d} x_{1} \int_{x_{c} / x_{1}}^{1} \mathrm{~d} x_{2} P\left(x_{1}\right) P\left(x_{2}\right) \\
& \times\left[\Theta(z-1) \Theta\left(\rho^{2}-z\right)+\Theta\left(z-x_{1}^{2}\right) \Theta\left(x_{1}^{2} \rho^{2}-z\right)\right] \\
& \times\left[\Theta(z-1) \Theta\left(\rho^{2}-z\right)+\Theta\left(z-x_{2}^{2}\right) \Theta\left(x_{2}^{2} \rho^{2}-z\right)\right] .
\end{aligned}
$$

We see that the leading contributions to $\Sigma^{2 \gamma}$ may be expressed in terms of kernels for the evolution equation for structure functions.

The functions $\phi^{\gamma \gamma}$ and $\phi_{\gamma}^{\gamma}$ in expression Eq. (28) collect the next-to-leading contributions which cannot be obtained by the structure functions method [18]. They have a form that can be obtained by comparing the results in the leading logarithmic approximation with the logarithmic ones given above.

\section{Pair production}

Pair production process in high-energy $\mathrm{e}^{+} \mathrm{e}^{-}$ collisions was considered about 60 years ago (see [13] and references therein). In particular it was found that the total cross-section contains cubic terms in large logarithm $L$. These terms come from the kinematics when the scattered electron and positron move in narrow (with opening angles $\sim m / \epsilon$ ) cones and the created pair have the invariant mass of the order of $m$ and moves preferably along either the electron beam direction or the positron one. According to the conditions of the LEP detectors, such a kinematics can be excluded. In the relevant kinematical region a parton-like description could be used giving $L^{2}$ and $L$-enhanced terms.

We accept the LEP 1 conventions whereby an event of the Bhabha process is defined as one in which the angles of the simultaneously registered particles hitting opposite detectors (see Eq. (48)).

The method, developed by one of us (N.P.M.) 16, 17, of calculating the real hard pair production cross-section within logarithmic accuracy (see the discussion in sect. 6) consists in separating the contributions of the collinear and semicollinear kinematical regions. In the first one (CK) we suggest that both electron and positron from the created pair go in the narrow cone around the direction of one charged particle [the projectile (scattered) electron $\boldsymbol{p}_{1}\left(\boldsymbol{q}_{1}\right)$ or the projectile (scattered) positron $\left.\boldsymbol{p}_{2}\left(\boldsymbol{q}_{2}\right)\right]$ :

$$
\begin{aligned}
& \widehat{\boldsymbol{p}_{+} \boldsymbol{p}_{-}} \sim \widehat{\boldsymbol{p}_{-} \boldsymbol{p}_{i}} \sim \widehat{\boldsymbol{p}_{+} \boldsymbol{p}_{i}}<\theta_{0} \ll 1, \\
& \varepsilon \theta_{0} / m \gg 1, \boldsymbol{p}_{i}=\boldsymbol{p}_{1}, \boldsymbol{p}_{2}, \boldsymbol{q}_{1}, \boldsymbol{q}_{2} .
\end{aligned}
$$

The contribution of the CK contains terms of order $(\alpha L / \pi)^{2},(\alpha / \pi)^{2} L \ln \left(\theta_{0} / \theta\right)$ and $(\alpha / \pi)^{2} L$, where $\theta=\widehat{\boldsymbol{p}}_{-} \boldsymbol{q}_{1}$ is the scattering angle. In the semi-collinear region only one of conditions (30) on the angles is fulfilled:

$$
\begin{aligned}
& \widehat{\boldsymbol{p}}_{+\boldsymbol{p}_{-}}<\theta_{0}, \quad{\widehat{\boldsymbol{\boldsymbol { p } _ { \pm }}}}_{i}>\theta_{0} ; \\
& \text { or } \quad{\widehat{\boldsymbol{p}} \boldsymbol{p}_{i}}_{i}<\theta_{0}, \quad{\widehat{\boldsymbol{p}}+\boldsymbol{p}_{i}}_{\boldsymbol{n}}>\theta_{0} \text {; } \\
& \text { or } \quad{\widehat{\boldsymbol{p}} \boldsymbol{p}_{i}}_{i}>\theta_{0}, \quad \widehat{\boldsymbol{p}+\boldsymbol{p}}_{i}<\theta_{0} \text {. }
\end{aligned}
$$

The contribution of the SCK contains terms of the form:

$$
\left(\frac{\alpha}{\pi}\right)^{2} L \ln \frac{\theta_{0}}{\theta}, \quad\left(\frac{\alpha}{\pi}\right)^{2} L .
$$

The auxiliary parameter $\theta_{0}$ drops out in the total sum of the CK and SCK contributions.

Taking into account the leading and next-toleading terms we can write the full hard pair contribution including also the pair emission along 
the positron direction, after the integration over $x_{2}$ as

$$
\begin{aligned}
\sigma_{\text {hard }} & =2 \frac{\alpha^{4}}{\pi Q_{1}^{2}} \int_{1}^{\rho^{2}} \frac{\mathrm{d} z}{z^{2}} \int_{x_{c}}^{1-\Delta} \mathrm{d} x\left\{L^{2}(1+\Theta) R(x)\right. \\
& \left.+\mathcal{L}\left[\Theta F_{1}(x)+F_{2}(x)\right]\right\} \\
F_{1,2}(x) & =d(x)+C_{1,2}(x) \\
d(x) & =\frac{1}{1-x}\left(\frac{8}{3} \ln (1-x)-\frac{20}{9}\right) \\
R(x) & =\frac{1}{3} \frac{1+x^{2}}{1-x}+\frac{1-x}{6 x}\left(4+7 x+4 x^{2}\right) \\
& +(1+x) \ln x
\end{aligned}
$$

where $C_{1}(x)$ and $C_{2}(x)$ are known functions [7].

Eq. (33) describes the small-angle high-energy cross-section for the pair production process, provided that the created hard pair can move along both electron and positron beam directions.

The contribution to the cross-section of the small-angle Bhabha scattering connected with the real soft (with energy lower than $\Delta \varepsilon$ ) and virtual pair production can be defined 21] by the formula:

$$
\begin{aligned}
\sigma_{\text {soft }+ \text { virt }} & =\frac{4 \alpha^{4}}{\pi Q_{1}^{2}} \int_{1}^{\rho^{2}} \frac{\mathrm{d} z}{z^{2}}\left\{L^{2}\left(\frac{2}{3} \ln \Delta+\frac{1}{2}\right)\right. \\
& +\mathcal{L}\left(-\frac{17}{6}+\frac{4}{3} \ln ^{2} \Delta\right. \\
& \left.\left.-\frac{20}{9} \ln \Delta-\frac{4}{3} \zeta_{2}\right)\right\} .
\end{aligned}
$$

Using Eqs. (33) and (34) it is easy to verify that the auxiliary parameter $\Delta$ is cancelled in the sum $\sigma_{\text {pair }}=\sigma_{\text {hard }}+\sigma_{\text {soft }+ \text { virt }}$. We can, therefore, write the total contribution $\sigma_{\text {pair }}$ as

$$
\begin{aligned}
& \sigma_{\text {pair }}=\frac{2 \alpha^{4}}{\pi Q_{1}^{2}} \int_{1}^{\rho^{2}} \frac{\mathrm{d} z}{z^{2}}\left\{L ^ { 2 } \left(1+\frac{4}{3} \ln \left(1-x_{c}\right)\right.\right. \\
& \left.-\frac{2}{3} \int_{x_{c}}^{1} \frac{\mathrm{d} x}{1-x} \bar{\Theta}\right)+\mathcal{L}\left[-\frac{17}{3}-\frac{8}{3} \zeta_{2}\right. \\
& -\frac{40}{9} \ln \left(1-x_{c}\right)+\frac{8}{3} \ln ^{2}\left(1-x_{c}\right)
\end{aligned}
$$

$$
\begin{aligned}
& \left.+\int_{x_{c}}^{1} \frac{\mathrm{d} x}{1-x} \bar{\Theta} \cdot\left(\frac{20}{9}-\frac{8}{3} \ln (1-x)\right)\right] \\
& +\int_{x_{c}}^{1} \mathrm{~d} x\left[L^{2}(1+\Theta) \bar{R}(x)+\mathcal{L}\left(\Theta C_{1}(x)\right.\right. \\
& \left.\left.\left.+C_{2}(x)\right)\right]\right\}, \\
& \bar{R}(x)=R(x)-\frac{2}{3(1-x)}, \quad \bar{\Theta}=1-\Theta .
\end{aligned}
$$

The right-hand side of Eq. (35) gives the contribution to the small-angle Bhabha scattering cross-section for pair production. It is finite and can be used for numerical estimations. The leading term can be described by the electron structure function $D_{e}^{\bar{e}}(x)$ [19].

\section{Terms of $\mathcal{O}(\alpha \mathcal{L})^{3}$}

In order to evaluate the leading logarithmic contribution represented by terms of the type $(\alpha \mathcal{L})^{3}$, we use the iteration up to $\beta^{3}$ of the master equation [18] obtained in Ref. [19]. To simplify the analytical expressions we adopt here a realistic assumption about the smallness of the threshold for the detection of the hard subprocess energy and neglect terms of the order of:

$x_{c}^{n}\left(\frac{\alpha}{\pi} \mathcal{L}\right)^{3} \leq 3 \cdot 10^{-5}, \quad n=1,2,3$.

We may, therefore, limit ourselves to consider the emission by the initial electron and positron. Three photons (virtual and real) contribution to $\Sigma$ have the form:

$$
\begin{aligned}
& \Sigma^{3 \gamma}=\frac{1}{4}\left(\frac{\alpha}{\pi} \mathcal{L}\right)^{3} \int_{1}^{\rho^{2}} \frac{\mathrm{d} z}{z^{2}} \int_{x_{c}}^{1} \mathrm{~d} x_{1} \int_{x_{c}}^{1} \mathrm{~d} x_{2} \Theta\left(x_{1} x_{2}-x_{c}\right) \\
& \times\left[\frac{1}{6} \delta\left(1-x_{2}\right) P^{(3)}\left(x_{1}\right) \Theta\left(x_{1}^{2} \rho^{2}-z\right)\right. \\
& \left.+\frac{1}{2 x_{1}^{2}} P^{(2)}\left(x_{1}\right) P\left(x_{2}\right) \Theta_{1} \Theta_{2}\right]\left(1+\mathcal{O}\left(x_{c}^{3}\right)\right) \\
& \Theta_{1} \Theta_{2}=\Theta\left(z-\frac{x_{2}^{2}}{x_{1}^{2}}\right) \Theta\left(\rho^{2} \frac{x_{2}^{2}}{x_{1}^{2}}-z\right)
\end{aligned}
$$


$P^{(3)}(x)$ is the three-loop splitting function. The contribution to $\Sigma$ of the process of pair production accompanied by photon emission when both, pair and photons, may be real and virtual has the form (with respect to paper by M. Skrzypek 19] we include also the non-singlet mechanism of pair production):

$$
\begin{aligned}
& \Sigma^{e^{+} e^{-} \gamma}=\frac{1}{4}\left(\frac{\alpha}{\pi} \mathcal{L}\right)^{3} \int_{1}^{\rho^{2}} \mathrm{~d} z z^{-2} \int_{x_{c}}^{1} \mathrm{~d} x_{1} \int_{x_{c}}^{1} \mathrm{~d} x_{2} \\
& \times \Theta\left(x_{1} x_{2}-x_{c}\right)\left\{\frac{1}{3}\left[R^{P}\left(x_{1}\right)-\frac{1}{3} R^{s}\left(x_{1}\right)\right]\right. \\
& \times \delta\left(1-x_{2}\right) \Theta\left(x_{1}^{2} \rho^{2}-z\right) \\
& \left.+\frac{1}{2 x_{1}^{2}} P\left(x_{2}\right) R\left(x_{1}\right) \Theta_{1} \Theta_{2}\right\}
\end{aligned}
$$

where

$$
\begin{aligned}
& R(x)=R^{s}(x)+\frac{2}{3} P(x) \\
& R^{s}(x)=\frac{1-x}{3 x}\left(4+7 x+4 x^{2}\right)+2(1+x) \ln x \\
& R^{P}(x)=R^{s}(x)\left(\frac{3}{2}+2 \ln (1-x)\right) \\
& +(1+x)\left(-\ln ^{2} x+4 \operatorname{Li}_{2}(1-x)\right. \\
& +\frac{1}{3}\left(-9-3 x+8 x^{2}\right) \ln x \\
& +\frac{2}{3}\left(-\frac{3}{x}-8+8 x+3 x^{2}\right)+\frac{2}{3} P^{(2)}(x)
\end{aligned}
$$

The quantity $\Sigma$ depends on the parameters $x_{c}, \rho$ and $Q_{1}^{2}$.

\section{Estimates of neglected terms and nu- merical results}

The uncertainty of our calculations is defined by neglected terms. Let us list them.

a) Terms of the first order RC coming from annihilation-type diagrams (15):

$$
\frac{\alpha}{\pi} \theta_{1}^{2} \int_{\theta_{1}^{2}}^{\theta_{2}^{2}} \frac{\mathrm{d} \theta}{\theta^{2}} \Delta_{\theta} \leq 0.10 \cdot 10^{-4} .
$$

b) Similar terms in the second order do not exceed (see sect. 4)

$$
\begin{aligned}
& \left(\frac{\alpha}{\pi}\right)^{2} \theta_{1}^{2} \int_{\theta_{1}^{2}}^{\theta_{2}^{2}} \frac{\mathrm{d} \theta}{\theta^{2}} l^{4} \leq 0.23 \cdot 10^{-4}, \\
& \left(\frac{\alpha}{\pi}\right)^{2}\left(\theta_{2}^{4}-\theta_{1}^{4}\right) \mathcal{L}^{4} \leq 0.5 \cdot 10^{-5} .
\end{aligned}
$$

c) We neglect terms which violate the eikonal approximation:

$\frac{\alpha}{\pi} \frac{Q^{2}}{s} \leq 0.3 \cdot 10^{-6}$.

d) We omit term of the second order which are not enhanced by large logarithms:

$\left(\frac{\alpha}{\pi}\right)^{2}=0.5 \cdot 10^{-5}$.

e) Creation of heavy pairs $(\mu \mu, \tau \tau, \pi \pi, \ldots)$ gives in sum at least one order of magnitude smaller than the corresponding contribution due to light particle production 22]:

$\Sigma_{\pi \pi}+\Sigma_{\mu \mu}+\Sigma_{\tau \tau} \leq 0.1 \Sigma^{e^{+} e^{-}} \leq 0.5 \cdot 10^{-4}$.

f) Higher-order corrections, including soft and collinear multi-photon contributions, can be neglected since they only give contributions of the type $(\alpha L / \pi)^{4} \leq 0.2 \cdot 10^{-5}$ or less.

g) The terms in the third order associated with the emission off the final particlest:

$x_{c}\left(\frac{\alpha \mathcal{L}}{\pi}\right)^{3} \leq 0.3 \cdot 10^{-4} \quad\left(\right.$ for $\left.x_{c}=0.5\right)$.

Regarding all the uncertainties a)-g) and (82) as independent ones we conclude the total theoretical uncertainty of our results to be $\pm 0.006 \%$.

Let us define $\Sigma_{0}^{0}$ to be equal to $\left.\Sigma_{0}\right|_{\Pi=0}$ (see Eq. (21)), which corresponds to the Born crosssection obtained by switching off the vacuum polarization contribution $\Pi(t)$. For the experimentally observable cross-section we obtain:

$$
\begin{aligned}
\sigma & =\frac{4 \pi \alpha^{2}}{Q_{1}^{2}} \Sigma_{0}^{0}\left(1+\delta_{0}+\delta^{\gamma}+\delta^{2 \gamma}+\delta^{e^{+} e^{-}}\right. \\
& \left.+\delta^{3 \gamma}+\delta^{e^{+} e^{-} \gamma}\right),
\end{aligned}
$$

${ }^{4}$ Usually, in a calorimetric experimental set-up such terms do not contribute. 
where

$\Sigma_{0}^{0}=\left.\Sigma_{0}\right|_{\Pi=0}=1-\rho^{-2}+\Sigma_{W}+\left.\Sigma_{\theta}\right|_{\Pi=0}$

and

$\delta_{0}=\frac{\Sigma_{0}-\Sigma_{0}^{0}}{\Sigma_{0}^{0}}, \delta^{\gamma}=\frac{\Sigma^{\gamma}}{\Sigma_{0}^{0}}, \delta^{2 \gamma}=\frac{\Sigma^{2 \gamma}}{\Sigma_{0}^{0}}, \cdots$.

The numerical results are presented in Table 1 . Each of the contributions to $\sigma$ has a sign that can change because of the interplay between real and virtual corrections. The cross-section corresponding to the Born diagrams for producing a real particle is always positive, whereas the sign of the radiative corrections depends on the order of perturbation theory. For the virtual corrections at odd orders it is negative, and at even orders it is positive. When the aperture of the counters is small the compensation between real and virtual corrections is not complete. In the limiting case of small aperture $\left(\rho \rightarrow 1, x_{c} \rightarrow 1\right)$ the virtual contributions dominate.

The numerical results were obtained by using the NLLBHA fortran code [23].

The approach described above to the small angle electron-positron cross-section can be also used to evaluate, with a next-to-next-to-leading accuracy, radiative corrections to the electron line at HERA in the small- $x$ region. This problem is under investigation.

The analytical and the numerical calculations for the cross-section in the non symmetrical Narrow-Wide configuration are in progress and will be presented elsewhere.

\section{Acknowledgements}

We thank Johannes Bluemlein for inviting us to this workshop. We are grateful for support to the Istituto Nazionale di Fisica Nucleare (INFN), to the International Association (INTAS) for the grant 93-1867 and to the Russian Foundation for Basic Research (RFBR) for the grant 96-0217512. One of us (L.T.) would like to thank H. Czyz, M. Dallavalle, B. Pietrzyk and T. Pullia for several useful discussions at various stages of the work and the CERN theory group for the hospitality. One of us (A.A.) is thankful to the Royal Swedish Academy of Sciences for an ICFPM grant.

\section{REFERENCES}

1. W. Beenakker, F.A. Berends and S.C. van der Marck, Nucl. Phys. B355 (1991) 281;

M. Cacciari, A. Deandrea, G. Montagna, O. Nicrosini and L. Trentadue, Phys. Lett. B271 (1991) 431;

M. Caffo, H. Czyz and E. Remiddi, Nuovo Cim. 105A (1992) 277;

K.S. Bjorkenvoll, G. Fäldt and P. Osland, Nucl. Phys. B386 (1992) 280, 303;

W. Beenakker and B. Pietrzyk, Phys. Lett. B296 (1992) 241; and B304 (1993) 366.

M. Cacciari, G. Montagna, O. Nicrosini and F. Piccinini, Comput. Phys. Commun. 90 (1995) 301.

2. S. Jadach et al., Phys. Rev. D47 (1993) 3733; S. Jadach, E. Richter-Was, B.F.L. Ward and Z. Was, Phys. Lett. B260 (1991) 438, and 268 (1991) 253;

S. Jadach, M. Skrzypek and B.F.L. Ward, Phys. Lett. B257 (1991) 173.

S. Jadach et al., Phys. Lett. B353 (1995) 362.

3. R. Budny, Phys. Lett. 55B (1975) 227;

D. Bardin, W. Hollik and T. Riemann, Z. Phys. C49 (1991) 485;

M. Boehm, A. Denner and W. Hollik, Nucl. Phys. B304 (1988) 687.

4. G. Altarelli, Lectures given at the E. Majorana Summer Scool, Erice, Italy, July 1993, CERN-TH.7072/93.

5. LEP Electroweak Working Group, A Combination of Preliminary LEP Electroweak Results from the 1995 Summer Conferences, 1995, CERN report LEPEWWG/95-02.

6. V.S. Fadin, E.A. Kuraev, L.N. Lipatov, N.P. Merenkov and L. Trentadue, Yad. Fiz. 56 (1993) 145;

S. Jadach et al., Phys. Lett. B253 (1991) 469.

7. A.B. Arbuzov, V.S. Fadin, E.A. Kuraev, L.N. Lipatov, N.P. Merenkov and L. Trentadue, preprint CERN-TH-95-313, to be published in Nucl. Phys. B.

8. R.V. Polovin, JEPT 31 (1956) 449 ;

F.A. Redhead, Proc. Roy. Soc. 220 (1953) 219

F.A. Berends et al., Nucl. Phys. B68 (1974) 
Table 1

The values of $\delta^{i}$ in per cent for $s^{1 / 2}=91.161 \mathrm{GeV}, \theta_{1}=1.61^{\circ}, \theta_{2}=2.8^{\circ}, \sin ^{2} \theta_{W}=0.2283, \Gamma_{Z}=2.4857$ $\mathrm{GeV}$.

\begin{tabular}{c|r|r|r|r|r|r|r|r|}
\hline$x_{c}$ & $\delta_{0}$ & $\delta^{\gamma}$ & $\delta_{\text {leading }}^{2 \gamma}$ & $\delta_{\text {nonlead }}^{2 \gamma}$ & $\delta^{e^{+}} e^{-}$ & $\delta^{e^{+} e^{-} \gamma}$ & $\delta^{3 \gamma}$ & $\sum \delta^{i}$ \\
\hline 0.1 & 4.120 & -8.918 & 0.657 & 0.162 & -0.016 & -0.017 & -0.019 & -4.031 \\
0.2 & 4.120 & -9.226 & 0.636 & 0.156 & -0.027 & -0.011 & -0.016 & -4.368 \\
0.3 & 4.120 & -9.626 & 0.615 & 0.148 & -0.033 & -0.008 & -0.013 & -4.797 \\
0.4 & 4.120 & -10.147 & 0.586 & 0.139 & -0.039 & -0.005 & -0.010 & -5.356 \\
0.5 & 4.120 & -10.850 & 0.539 & 0.129 & -0.044 & -0.003 & -0.006 & -6.115 \\
0.6 & 4.120 & -11.866 & 0.437 & 0.132 & -0.049 & -0.002 & -0.001 & -7.229 \\
0.7 & 4.120 & -13.770 & 0.379 & 0.130 & -0.057 & -0.001 & 0.005 & -9.194 \\
0.8 & 4.120 & -17.423 & 0.608 & 0.089 & -0.069 & 0.001 & 0.013 & -12.661 \\
0.9 & 4.120 & -25.269 & 1.952 & -0.085 & -0.085 & 0.005 & 0.017 & -19.379 \\
\hline
\end{tabular}

541.

9. S. Eidelman, F. Jegerlehner, Z. Phys. C67 (1995) 585.

10. E.A. Kuraev, L.N. Lipatov and N.P. Merenkov, Phys Lett. 47B (1973) 33; preprint 46 LNPI, 1973;

H. Cheng, T. T. Wu, Phys. Rev. 187 (1969) 1868 ;

V.S. Fadin, E.A. Kuraev, L.N. Lipatov, N.P. Merenkov and L. Trentadue, Yad. Fiz. 56 (1993) 145.

11. G. Fäldt and P. Osland, Nucl. Phys. B413 (1994) 16; Erratum ibidem B419 (1994) 404.

12. D.R. Yennie, S.C. Frautchi, H. Suura, Ann. Phys. 13 (1961) 379.

13. V.N. Baier, V.S. Fadin, V. Khoze and E.A. Kuraev, Phys. Rep. 78 (1981) 294;

V.M. Budnev, I.F. Ginzburg, G.V. Meledin and V.G. Serbo, Phys. Rep. C15 (1975) 183.

14. E.A. Kuraev, N.P. Merenkov, V.S. Fadin, Sov. J. Nucl. Phys. 45 (1987) 486.

15. H. Cheng and T.T. Wu, Expanding Protons: Scattering at High Energies, London, England, 1986.

16. N.P. Merenkov, Sov. J. Nucl. Phys. 48 (1988) 1073.

17. N.P. Merenkov, Sov. J. Nucl. Phys. 50 (1989) 469.

18. L.N. Lipatov, Sov. J. Nucl. Phys. 20 (1974) 94;

G. Altarelli and G. Parisi, Nucl. Phys. B126 (1977) 298;

E. A. Kuraev and V. S. Fadin, Sov. J. of
Nucl. Phys. 41 (1985) 466; Preprint INP 8444, Novosibirsk, 1984;

O. Nicrosini and L. Trentadue, Phys. Lett. 196B (1987) 551.

19. M. Skrzypek, Acta Phys. Pol. B23 (1993), 135

E.A. Kuraev, N.P. Merenkov and V.S. Fadin, Sov. J. Nucl. Phys. 47 (1988) 1009.

S. Jadach, M. Skrzypek, B.F.L. Ward, Phys. Lett. B257 (1991) p.173.

20. A.B. Arbuzov, E.A. Kuraev, N.P. Merenkov and L. Trentadue, JETP 108 (1995) 1164; preprint CERN-TH/95-241, JINR E2-95110, Dubna, 1995.

21. A.B. Arbuzov, V.S. Fadin, E.A. Kuraev, L.N. Lipatov, N.P. Merenkov and L. Trentadue, Report CERN 95-03 (1995) p. 369.

22. V.N. Baier, V.S. Fadin, V.M. Katkov, Emission of relativistic electrons, Moscow, Atomizdat, 1973.

23. A short write-up of the NLLBHA code can be found in the CERN Yellow Report CERN-9601, vol.2. A copy of the program is available, upon request, from the authors. 